(Aus dem pharmakologischen Institut der Reichsuniversität Utrecht.)

\title{
Welche Teile des Zentralnervensystems müssen für das Zustandekommen der tonischen Hals- und Labyrinthreflexe auf die Körper- muskulatur vorhanden sein?
}

Von

R. Magnus.

(Mit 3 Textfiguren.)

\section{Einleitung.}

Die tonischen Reflexe, durch welche bei einer bestimmten gegebenen Kopfstellung eine gesetzmässig zugehörige Körperstellung zustande gebracht wird, sind in einer Reihe von vorhergehenden Abhandlungen ${ }^{1}$ ) nach verschiedenen Richtungen durchuntersucht worden. Die Muskeln, die sich an diesen Reflexen beteiligen, die Rezeptoren, die sie auslösen, die afferenten Bahnen, welche die Impulse dem Zentralnervensystem zuführen, die Gesetze, nach denen sich die verschiedenen Reflexe addieren und superponieren, die Rolle, welche sie bei den normalen und pathologischen Bewegungen und Stellungen der Tiere spielen, sind in den Hauptzügen festgestellt. Es erübrigt noch, diejenigen Teile des Zentralnervensystems zu ermitteln, deren Anwesenheit für das Zustandekommen dieser Reflexe unumgänglich notwendig ist. Es ist die Aufgabe der vorliegenden Untersuchung, diese Lücke auszufüllen.

1) R. Magnus und A. de Kleijn, Pflüger's Arch. Bd. 145 S. 455. 1912, Bd. 147 S. 403. 1912, Bd. 154 S. 163.1913 , Bd. 154 S. 178. 1913. Münch. med. Wochenschr, 1913 S. 2566. - W. Weiland, Pflüger's Arch. Bd. 147 S. 1. 1912. - R. Magnus und C. G. L. Wolf, Pflüger's Arch. Bd. 149 S. 447. 1913. - R. Magnus und W. Storm van Leeuwen, Pflüger's Arch. Bd. 159 S. 157. 1914. - A. de Kleijn, Pflüger's Arch. Bd. 159 S. 218. 1914. - C. Socin und W. Storm van Leeuwen, Pflüger's Arch. Bd. 159 s. 251. 1914. 
Es handelt sich um zwei Gruppen von tonischen Reflexen. Die erste wird ausgelöst durch Änderungen der Stellung des Koples im Raume, nimmt ihren Ursprung in den Labyrinthen und wird dem Zentralorgan durch die Octavi zugeleitet. Die zweite wird ausgelöst durch Änderung der Stellung des Kopfes zum Rumpfe, nimmt ihren Ursprung in den Muskeln, Sehnen oder Gelenken des Halses und wird dem Zentrum (bei der Katze) im wesentlichen durch die drei obersten cervicalen Hinterwurzeln zugeleitet. Beide Gruppen von Reflexen lassen sich nicht nur am intakten Tier, sondern in besonders deutlicher Weise nach dem Dezerebrieren nachweisen. Also genügen, falls man von den Reflexen auf die Augenmuskeln abstrahiert, die Anwesenheit des Rückenmarkes, der Medulla oblongata, der Brücke, des Kleinhirns und der hinteren Vierhügel für das Zustandekommen der Reflexe.

In einer ersten Reihe der in dieser Arbeit geschilderten Versuche wurde bei dezerebrierten Katzen das Kleinhirn exstirpiert. Es stellte sich heraus, dass danach sowohl die Hals- wie die Labyrinthreflexe noch unverändert auszulösen waren. Darauf wurde in einer zweiten Versuchsreihe nach Entfernung des Cerebellum der Hirnstamm von den Vierhügeln ab kaudalwärts durch eine Reihe sich folgender Frontalschnitte schrittweise abgetragen und festgestellt, nach welchem Frontalschnitt die Labyrinthreflexe und nach welchem Schnitte die Halsreflexe aufhörten.

Über das Ergebnis von im ganzen 23 nach diesem Plane ausgeführten Versuchen an dezerebrierten Katzen soll im folgenden berichtet werden.

\section{Methodik.}

Die Katzen wurden in tiefer Äthernarkose tracheotomiert und die Äthernarkose danach mittelst der künstlichen Atmung fortgesetzt. Nach Unterbindung beider Karotiden und doppelseitiger Vagotomie wurde das Rückenmark am zwölften Brustwirbel provisorisch freigelegt, um, falls die Untersuchung allein auf die Reflexe an den Vorderbeinen beschränkt werden sollte, später das Rückenmark schnell und ohne Störung durchschneiden zu können. Die Rückenwunde wurde durch Klammern verschlossen.

Darauf wurde die Kopfhaut über dem Scheitel durch einen Längsschnitt gespalten, beide Temporalmuskeln zurückpräpariert und die Muskelansätze am Planum occipitale bis herunter an die Mem- 
brana obturatoria abgelöst, so dass das ganze Schädeldach vom Hinterhauptsloch bis zu den Augen freilag. Nunmehr wurde auf beiden Plana temporalia je ein Trepanationsloch angebracht. Die ganze folgende Operation wurde nun wesentlich erleichtert durch Anwendung der von Sherrington auf dem Groninger Physiologenkongress demonstrierten Methode der temporären Abklemmung der Vertebralarterien zwischen Atlas und Epistropheus, die mit Daumen und Zeigefinger leicht ausführbar ist. Hierdurch wird erreicht, dass man die ganze folgende intrakranielle Operation (wenigstens bei kleinen und mittelgrossen Katzen) ohne jede Blutung vornehmen kann. Nur bei sehr grossen Katzen mit dicken Halsmuskeln ist die Kompression der Vertebrales schwieriger und oft nicht imstande, die Blutung ganz zu verhindern.

Nunmehr wurde das Schädeldach über dem Grosshirn mit der Knochenzange entfernt, die Dura breit gespalten, der Hirnstamm in der Ebene des knöchernen Tentorium cerebelli mit einem Spatel quer durchtrennt und das ganze Gehirn vor den hinteren Vierhügeln in toto ausgeräumt. Dabei tut man gut, die Dura an der Sebädelbasis nicht abzulösen, da sonst leicht nach Beendigung der Operation eine Nachblutung aus den durchrissenen arteriellen Gefässen erfolgt.

Die Narkose wird jetzt abgestellt und bei fortdauernder Kompression der Vertebrales das Tentorium cerebelli und die Sehädeldecke über dem Kleinhirn bis zum Hinterhauptsloch abgetragen. Seitlich geht man dabei bis zur Felsenbeinpyramide. Das Kleinhiru liegt jetzt vollständig frei. Der vordere Pol des Wurmes wird durch vorsichtiges Zerreissen der Pia-Arachnoides von den hinteren Vierhügeln abgelöst und ein stumpfer Finder unter dem Kleinhirn nach hinten geschoben, bis derselbe am Calamus scriptorius dorsal von der Medulla oblongata wieder zum Vorschein kommt.

Zur Entfernung des Kleinhirns bin ich auf verschiedene Weise vorgegangen. Entweder man geht neben dem Finder mit einem schmalen Messer ein und trägt durch zwei horizontale Schnitte nach rechts und links den Wurm und die dorsalen Partien der Seitenteile ab. Danach werden dann die übrigbleibenden ventralen Partien der Seitenteile entfernt. Dieses Verfahren ist sehr schonend; man lässt aber dabei gewöhnlich mehr von den Kleinhirnstielen stehen als bei den anderen Methoden. - Daher wurde in einer Reihe von Versuchen das Kleinhirn auf dem untergeschobenen Finder in die Höhe gehoben, bis man die Kleinhirnstiele sich anspannen sieht. Diese 
werden dann vom vierten Ventrikel aus mit dem Messer durchtrennt. Hierbei entfernt man also das Kleinhirn in einem Stück und erbält daher anatomisch das beste Resultat. Beim Anheben des Kleinhirns werden aber die Stiele mehr oder weniger gezerrt, und es ist dieses wahrscheinlich der Grund, weshalb bei den in dieser Weise operierten Tieren die Enthirnungsstarre sich nicht so kräftig entwickelte und die tonischen Reflexe nicht so lebhaft waren wie in den übrigen Experimenten. - Daher wurde in den späteren Versuchen zunächst auf dem Finder eine Längsspaltung des Cerebellum vorgenommen, die beiden Hälften nach rechts und links herübergeklappt und darauf die gut sichtbaren Stiele von der Medialseite her mit dem Messer durchtrennt. Dieses Verfahren ist schonend und gibt anatomisch sehr gute Resultate.

Bei diesem Vorgehen bleiben beide Octavi sowie die Trigemini und Faciales unberührt. Die lateralen Teile der Felsenbeinpyramiden liegen frei. Der Boden des vierten Ventrikels ist vollständig zu übersehen.

Fig. 1 gibt eine stereoskopische Aufnahme des nach der Dezerebrierung stehenbleibenden Hirnstammes mit intaktem Kleinhirn, Fig. 2 (S. 237) das bei Versuch 23 gewonnene Präparat samt dem in zwei Teilen exstirpierten Kleinhirn wieder.

Fig. 1.
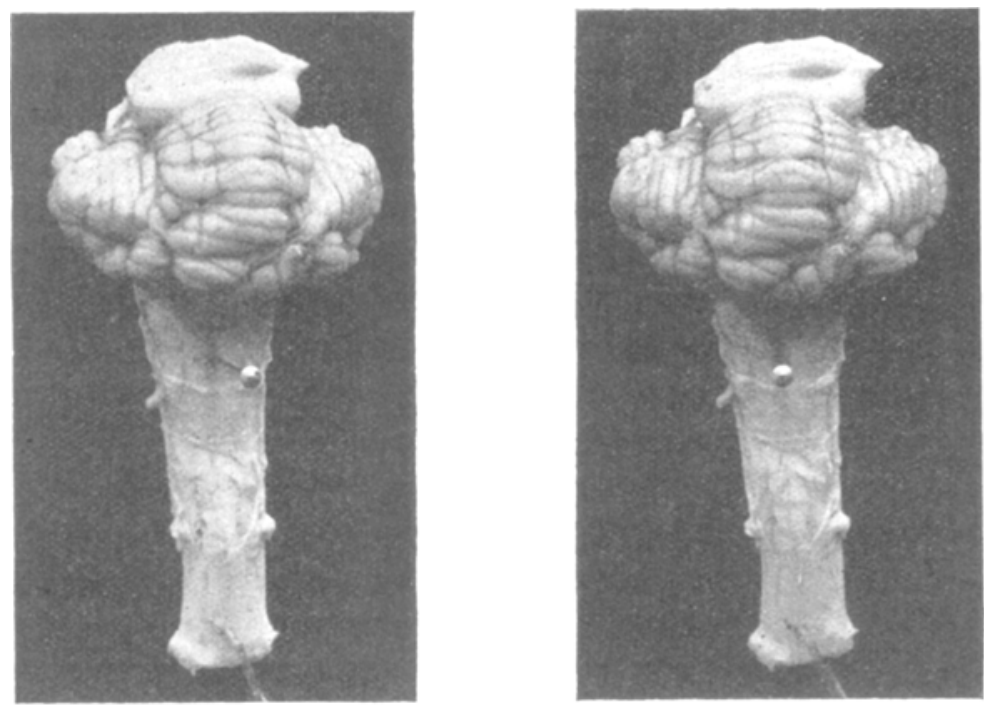
Die Dauer der ganzen Operation vom ersten Hautschnitt an beträgt 15-20 Minuten, die der eigentlichen Kleinhirnexstirpation 5-8 Minuten.

Die temporäre Kompression der Vertebralarterien wird nun beendet. Die nachfolgende Blutung wird dadurch vermindert, dass man das Tier für 15-20 Minuten mit boch erhobenem Kopf lagert. $\mathrm{Da}$ die ganze Schädelhöhle leer ist, führen Blutgerinnsel nicht leicht zu Kompressionserscheinungen. Tatsächlich war nur in einem Versuche eine geringere Kompression dureh Blutkoagula eingetreten. Die Haut wird über der Schädelöffnung durch Klammern geschlossen.

Erstaunlich ist, dass nach der eingreifenden Operation in der unmittelbaren Nähe der Medulla oblongata der Shock sebr gering ist. In der Mehrzahl der Fälle atmen die Tiere bereits am Ende der Operation spontan. Die Enthirnungsstarre der Vorderund oft auch der Hinterbeine beginnt sich sofort zu entwickeln und ist nach $1 / 4$ Stunde gewöhnlich sebr kräftig ausgesprochen. Gleichseitige und gekreuzte Reflexe an den Extremitäten sind stets, Corneal- und Ohrreflex häufig nach wenigen Minuten auszulösen. - Es gilt also auch für Operationen in der Nähe der Medulla oblongata die von Sherrington gefundene Regel, dass, wenn sie nach dem Dezerebrieren vorgenommen werden, kein oder nur ein minimaler Shock erfolgt.

In einer Reihe von Versuchen wurde, wie erwähnt, danach der Hirnstamn durch sukzessive Frontalschnitte von vorne nach hinten abgetragen. Dabei wurden jedesmal wieder die Vertebrales temporär abgeklemmt und etwaige Blutkoagula vorsichtig entfernt. Man kann sich dann mit Leichtigkeit anatomisch orientieren, die Entfernung des Schnittes von den Vierhügeln, dem Calamus scriptorius usw. mit dem Millimetermaassstab abmessen, die Vollständigkeit der Durchschneidung kontrollieren usw. Auch der Octavus lässt sich unter Leitung des Auges durchschneiden. Für Frontalschnitte im Bereiche des Halsmarkes werden die Dorsalteile der obersten Halswirbel mit der Knochenzange entfernt.

Wenn man den Hirnstamm dureh eine Reihe von Frontalschnitten immer mehr abtrennt, so nimmtallmählich die Enthirnungsstarre an Intensität ab. Für die Prüfung der Hals- und Labyrinthreflexe an den Gliedermuskeln ist es aber wünschenswert, dass die Muskeln sich in Zustande

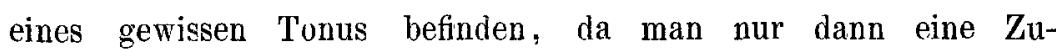
oder Abnahme des Tonus mit Sicherheit beurteilen kann. Es 
war daher für die Zwecke der vorliegenden Untersuchung von grossem Vorteil, dass jeder Frontalschnitt selbst als Reiz wirkt und für einige Zeit eine Zunahme des Tonus der Extremitätenmuskeln veranlasst. Da auch diese Frontalschnitte am dezerebrierten Präparat so gut wie keinen Shock veranlassen, so kann man diese Tonuszunahme benutzen, um schnell die An- oder Abwesenheit von Hals- und Labyrinthreflexen festzustellen. Aus diesem Grunde habe ich, wenn die Schnitte einmal in die hintere Hälfte der Rautengrube gekommen waren, niemals längere Pausen als etwa 5 Minuten zwischen den einzelnen Schnitten gemacht. Dann ist der Gliedertonus hinreichend, um das Vorhandensein tonischer Reflexe erkennen zu können.

Am Schluss jedes Versuches wurde die Sektion ausgeführt und das restierende Stück des Hirnstammes samt allen abgetrennten Stücken sowie das exstirpierte Kleinhirn in Formol gelegt. Die Präparate von den entscheidenden Versuchen habe ich Professor C. Winkler in Amsterdam vorgelegt, der soeben mit der Herausgabe eines anatomischen Atlas des Katzengehirnes beschäftigt ist und mir auf Grund seiner grossen Erfahrung genauere Angaben über die Lage der Schnitte und die Ausdehnung der exstirpierten Hirnteile machen konnte. Diese Mitteilungen, für die ich Herrn Winkler auch an dieser Stelle herzlich danke, sind im folgenden mit verwertet worden ${ }^{1}$ ).

Die Prüfung der Katzen auf das Vorhandensein von Hals- und Labyrinthreflexen geschah in der früher ausfübrlich geschilderten Weise. Wie man bei der Katze Hals- und Labyrinthreflexe am einfachsten voneinander unterscheiden kann, ist in Pflüger's Arch. Bd. 145 S. 514 ff. 1912 genauer angegeben worden. Labyrinthreflexe treten ein, wenn der Kopf seine Lage im Raume ändert, und zwar nur, wenn die Ebene der Mundspalte ihren Winkel mit der Horizontalebene irgendwie ändert. Es gibt nur eine Lage des Kopfes im Raume, bei der der Strecktonus der Extremitäten und der Tonus der Nackenheber maximal, und eine Lage, bei der er minimal ist (Maximum: wenn Scheitel unten und Mundspalte $45^{\circ}$ gegen die Horizontale gehoben; Minimum: wenn Scheitel oben und Mundspalte $45^{\circ}$ unter die Horizontale gesenkt). Bei den Labyrinthreflexen reagieren die vier Extremitäten stets gleichsinnig.

1) Prof. Winkler hat auch die mikroskopische Untersuchung der Präparate von zwei Versucken freundlichst übernommen. 
Die Halsreflexe werden ausgelöst durch Änderung der Stellung des Kopfes zum Rumpf. Bei Drehen und Wenden des Kopfes erfolgt Zunahme des Strecktonus in den "Kieferbeinen" und Abnahme des Strecktonus in den "Schädelbeinen". Bei Heben des Kopfes werden die Vorderbeine gestreckt und die Hinterbeine gebeugt, bei Senken des Kopfes die Vorderbeine gebeugt und die Hinterbeine gestreckt.

Da ein und dieselbe Kopf bewegung (Drehen, Wenden, Heben, Senken) bei den verschiedenen Lagen des Tieres im Raume (Fussstellung, Rückenlage, Seitenlage; Hängelage mit Kopf unten) zu den gleichen Halsreflexen, aber stets zu verschiedenen Labyrinthreflexen Anlass geben muss, so lässt sich durch Untersuchung des Einflusses der Kopfstellung bei verschiedenen Lagen des Tieres leicht ein sicheres Urteil darüber gewinnen, ob es sich im Einzelfalle um Halsoder Labyrinthreflexe oder um eine Kombination von beiden handelt.

Zur Erleichterung des Verständnisses wird in den unten aufgefübrten Versuchsprotokollen jedesmal angegeben werden, ob es sich um Hals- oder Labyrinthreflexe handelt. Für die Begründung wird auf die früheren Arbeiten verwiesen.

Wenden ist eine Bewegung des Kopfes um die Achse: ScheitelSchädelbasis; Drehen eine Bewegung um die Achse: SchnauzeHinterhauptsloch. Hehen und Senken erfolgen um die Bitemporalachse.

\section{Versuchsergebnisse.}

\section{a) Hals- und Labyrinthreflexe nach Exstirpation des Kleinhirns.}

Dass nach Entfernung des Kleinhirns bei der dezerebrierten Katze sowohl die tonischen Hals- als die Labyrinthreflexe unverändert erhalten sind, wird durch nachstehende Versuehsprotokolle bewiesen:

Versuch V. 26. Januar 1914.

Katze. Äthernarkose. Karotiden abgebunden, Vagi durehsehnitten. Freilegung des Rückenmarkes am zwölften Brustwirbel. Freilegung des Schädeldaches, doppelseitige Trepanation, Abklemmen der Arteriae vertebrales, Dezerebrieren mit Ausräumung des ganzen Gehirnes vor den Vierhügeln, Exstirpation des Kleinhirns. Der vierte Ventrikel liegt in ganzer Ausdehnung frei.

9 Uhr 45 Min. Ende der Operation, die im ganzen 20 Minuten gedauert hat. Spontanatmung. Sofort beginnende Starre der vier Beine.

10 Uhr 5 Min. Guter Zustand des Tieres.

In rechter Seitenlage bewirkt Kopfdrehen mit dem Scheitel nach 
unten starke Zunahme des Strecktonus im linken (oberen) Bein, während der Tonus des rechten (unteren) Beines ungeändert bleibt. Kopfdrehen mit dem Scheitel nach oben bewirkt Abnahme des Strecktonus im linken (oberen) Bein, keine Veränderung im rechten (unteren) Bein.

In linker Seitenlage treten auf Kopfdrehen nur Veränderungen im Strecktonus des rechten (oberen) Beines ein, während der Tonus im linken (unteren) Bein ungeändert bleibt (Hals- und Labyrinthreflexe gleich stark entwickelt).

10 Uhr 10 Min. Bei Kopfdrehen in Seitenlage werden bei Scheitel-unten beide Beine gestreckt, bei Scheitel-oben beide Beine gebeugt; das obere Bein reagiert aber viel stärker als das untere (Hals- und Labyrinthreflexe, letztere überwiegen).

10 Uhr 15 Min. Rückenlage, Kopfheben und -senken:

Die Vorderbeine haben maximalen Strecktonus, wenn die Schnauze $45^{\circ}$ über die Horizontale gehoben ist. Der Strecktonus sinkt deutlich, wenn der Kopf dorsalwärts gebeugt und die Schnauze dabei unter die Horizontale gesenkt wird. Der Strecktonus sinkt maximal, wenn der Kopf stark ventral gebeugt wird, bis die Schnauze zwischen den Vorderbeinen liegt (Labyrinthreflexe).

Rückenlage, Kopfdrehen: Tonuszunahme im Kieferbein, Abnahme im Schädelbein (Halsreflexe).

Rückenlage, Kopfwenden: Tonuszunahme im Kieferbein, Abnahme im Schädelbein (Halsreflexe).

Fussstellung, Kopfheben und -senken: Schwach wirksam.

Fussstellung, Kopfdrehen bei vertikal erhobener Schnauze: Kieferbein Tonuszunahme, Schädelbein Tonusabnahme (Halsreflexe).

Wird das ganze Tier, ohne die Stellung des Kopfes zum Rumpfe zu ändern, aus der Seitenlage in Rückenlage gedreht, so erfolgt eine beträchtliche Zunahme des Strektonus der Vorderbeine (Labyrinthreflex).

Hängelage, Kopf unten, Kopfheben und -senken: Wenn die Schnauze senkrecht nach unten hängt, ist der Strecktonus der Vorderbeine gering. Wird der Kopf stark ventralwärts gebeugt, so dass die Schnauze zwischen den Vorderbeinen steht und die Mundspalte über die Horizontale gehoben ist, so werden die Vorderbeine aktiv gestreckt (Labyrinthreflex).

Seitenlage, Vertebra-prominens-Reflex deutlich.

10 Uhr 25 Min. Durchschneidung des Rückenmarkes am zwölften Brustwirbel. 
10 Uhr $40 \mathrm{Min}$. Nochmalige gründliche Untersuchung auf Halsund Labyrinthreflexe mit demselben Ergebnis wie 10 Uhr 15 Min. Ausserdem werden noch folgende Reaktionen festgestellt:

Fussstellung, Kopfheben bewirkt Zunahme, Kopfsenken Abpahme des Strecktonus der Vorderbeine (Hals- und Labyrinthreflexe).

Fussstellung, Kopfwenden bei horizontaler Mundspalte: Kieferbein Tonuszunahme, Schädelbein Tonusabnahme (Halsreflex). Sektion: Der Dezerebrierungsschnitt geht durch das hintere Drittel der vorderen Vierhügel. Rechts ist etwas vom Hirnschenkel stehengeblieben. Der vierte Ventrikel liegt vollständig frei. Das Kleinhirn ist einschliesslich der Seitenteile entfernt. Die Kleinhirnstiele sind als Stümpfe noch so weit stehengeblieben, dass ein Teil der Nuclei dentati vielleicht noch erhalten ist (W inkler). Beide Octavi intakt.

Ergebnis: Bei einer dezerebrierten Katze wird das Kleinhirn bis auf die Kleinhirnstiele total entfernt. Danach sind durch Änderung der Kopfstellung sehr kräftige Hals- und Labyrinthreflexe auf die Muskulatur der Vorderbeine auszulösen. Die Enthirnungsstarre ist sehr hochgradig.

\section{Versuch VI. 27. Januar 1914.}

Katze. Äthernarkose. Operation wie in Versuch V. Exstirpation des KJeinhirns. Dauer der ganzen Operation 20 Minuten.

9 Uhr 50 Min. Ende der Operation. Spontanatmung. Sofort Starre aller vier Beine.

10 Ubr 12 Min. Das Tier kann tadellos auf seinen vier Beinen stehen und den Kopf frei heben.

Rechte und linke Seitenlage, Kopfdrehen: Bei "Scheitel-unten" Tonuszunahme, bei "Scheitel-oben" Tonusabnahme der Streckmuskeln der Vorderbeine. Das obenliegende Bein reagiert etwas stärker (Hals- und Labyrinthreflexe, letztere überwiegen).

Seitenlage, Kopfwenden: Dasselbe.

Seitenlage, Kopfheben und -senken: Dorsalbeugen des Kopfes bewirkt Tonuszunahme, Ventralbeugung bewirkt Tonusabnahme der Vorderbeinstrecker (reiner $\mathrm{H}$ als reflex).

Rückenlage, Kopfheben und -senken: Die Vorderbeine haben maximalen Strecktonus, wenn die Schnauze $45^{\circ}$ über die Horizontale gehoben ist. Der Tonus sinkt wenig, wenn der Kopf dorsalwärts gebeugt wird. Er sinkt maximal, wenn der Kopf so stark ventral 
gebeugt wird, dass sich die Schnauze zwischen den Vorderbeinen befindet (Hals - und Labyrinthreflexe).

Rückenlage, Kopfwenden: Kieferbein Tonuszunahme, Schädelbein Tonusabnabme (Halsreflex).

Rückenlage, Kopfdrehen: An beiden Vorderbeinen Tonusabnahme (Labyrinthreflex). An den Hinterbeinen Tonusabnahme im Schädelbein und Tonuszunahme im Kieferbein (Halsreflex).

Fussstellung, Kopfheben und -senken: Kopfheben bewirkt Tonuszunahme der Vorderbeine und Tonusabnahme der Hinterbeine. Kopfsenken bewirkt Tonusabnahme der Vorderbeine und Tonuszunahme der Hinterbeine (überwiegend Halsreflexe).

Fussstellung, Kopfwenden bei horizontaler Mundspalte: Tonuszunahme im vorderen Kieferhein, Tonusabnahme im vorderen Schädelbein (H als reflex).

Fussstellung, Kopfdrehen bei senkrecht erhobener Schnauze: An den Vorder- und Hinterbeinen Tonuszunahme in den Kieferbeinen, Tonusabnahme in den Schädelbeinen (Halsreflex).

Hängelage mit Kopf unten, Kopfheben und -senken: Alle vier Beine haben am wenigsten Strecktonus, wenn die Schnauze nach unten hängt. Sie strecken sich, wenn der Kopf so stark dorsal oder ventral gebeugt wird, dass die Mundspalte über die Horizontale gehoben wird (Labyrinthreflexe auf alle vier Beine).

10 Uhr 35 Min. Durchschneidung des Rückenmarkes am zwölften Brustwirbel. Starke Starre der Vorderbeine. Sehr gute spontane Atmung. Ohrreflex und Lidreflex vorhanden.

10 Uhr 45 Min. Nochmalige Prüfung des Einflusses der Kopfstellung auf den Tonus der Vorderbeine. Sehr starke Labyrinthreflexe.

Sektion: Dezerebrierung vollständig, gerade vor den hinteren Vierhügeln. Der vierte Ventrikel liegt vollständig frei. Das Kleinhirn ist einschliesslich der Seitenteile entfernt. Links sind auch die Kleinhirnstiele vollständig fortgenommen, rechts steht von den Stielen noch ein Stumpf, so dass auf der rechten Seite der Nucleus dentatus vielleicht teilweise erhalten ist (W inkler). Beide Octavi, Faciales und Trigemini intakt.

Ergebnis: Bei einer dezerebrierten Katze wird das Kleinhirn bis auf einen Stumpf des rechten Kleinhirnstieles total entfernt. Danach sind durch Änderung der Kopfstellung sehr kräftige Hals- und Labyrinthreflexe auf die Muskulatur aller vier Beine auszulösen. Die Enthirnunysstarre ist sehr hochgradig. 
Noch in einem weiteren Versuch (Nr. VIII vom 29. Januar 1914), in dem das Kleinhirn bis auf die Kleinhirnstiele total entfernt worden war, liessen sich sehr kräftige Hals- und Labyrinthreflexe auf die Vorder- und Hinterbeine nachweisen. Die ausführliche Wiedergabe des Protokolles erübrigt sich, da es fast vollständig mit dem von Versuch VI übereinstimmt.

\section{Versuch XVII. 16. Februar 1914.}

Katze. Äthernarkose. Karotiden abgebunden, Vagi durchtrennt. Freilegung des Rückenmarkes am zwölften Brustwirbel. Dezerebrierung unter Abklemmung der Vertebralarterien. Totalexstirpation des Kleinhirns.

9 Uhr 45 Min. Ende der Operation. Spontanatmung. Gute Starre der vier Extremitäten.

10 Uhr 20 Min. Sehr guter Zustand. Starre aller vier Beine. Obrreflex und Lidreflex stark vorhanden.

Seitenlage, Kopfdrehen: Bei "Scheitel-unten" starke Zunahme, bei "Scheitel-oben" starke Abnahme des Strecktonus beider Vorderbeine (sehr starke Labyrinthreflexe).

Seitenlage, Kopfwenden: Dasselbe (Laby rinthreflexe).

Seitenlage, Kopfheben und -senken: Dorsalbeugen des Kopfes bewirkt Zunahme, Ventralbeugen Abnahme des Strecktonus der Vorderbeine (Hals reflex).

Seitenlage, Vertebra-prominens-Reflex deutlich.

Rückenlage, Heben und Senken: Minimum des Strecktonus der Vorderbeine, wenn die Schnauze zwischen den Vorderbeinen, Maximum, wenn Schnauze $45^{\circ}$ über die Horizontale gehoben und wenn durch Dorsalbeugen des Kopfes unter die Horizontale gesenkt (Halsund Labyrinthreflexe).

Rückenlage, Kopfwenden: Tonuszunahme im Kieferbein, Tonusabnahme im Schädelhein (Halsreflexe).

Rückenlage, Kopfdrehen: Tonusabnahme beider Vorderbeine (Labyrinthreflexe).

Fussstellung, Kopfheben und -senken: Kopfheben bewirkt Streckung, Kopfsenken Beugung der Vorderbeine (Hals- und Labyrinthreflexe).

Fussstellung, Kopfdrehen bei erhobener Schnauze: Tonuszunahme im Kieferbein, Abrahme im Schadelbein (Halsreflex). 
Fussstellung, Kopfwenden bei horizontaler Mundspalte: Geringere Tonuszunahme im Kieferbein und Abnahme im Schädelbein (Hals reflexe).

Hängelage mit Kopf-unten, Kopfheben und -senken: Strecktonus der Vorderbeine minimal, wenn die Schnauze nach unten hängt, nimmt zu, wenn sie durch Dorsalbeugen, und wird maximal, wenn sie durch Ventralbeugen über die Horizontale geschoben wird (Labyrinthreflexe).

Beim Umlegen des ganzen Tieres aus der Fussstellung in Rückenlage, wobei die Stellung des Kopfes gegen den Rumpf nicht geändert wird, kommt es zu sehr starker Zunahme des Tonus der Vorderbeinstrecker und der Nackenheber (Labyrinthreflexe).

10 Uhr 50 Min. Durchtrennung des linken Nervus octavus. Darauf ist das linke Vorderbein schlaffer als das rechte. Bei Hängelage mit Kopf-unten ist der Kopf $45^{\circ}$ nach links gedreht. Die obere Thoraxapertur ist $30^{\circ}$, die untere Thoraxapertur $20^{\circ}$ gegen das Becken nach links gedreht. Bei Fussstellung ist der Kopf nach links gewendet. Zum Geradesetzen des Kopfes muss man bei Rückenlage einen grösseren muskulären Widerstand überwinden als bei Fussstellung (Labyrinthreflexe).

Beim Umlegen aus der Fussstellung in Rückenlage kommt es zu deutlicher Tonuszunahme der Vorderbeine und Retraktion des (gedrehten) Nackens (L a by rin threflexe).

Auch sonst lassen sich bei Drehen, Wenden, Heben und Senken des Kopfes in den _verschiedenen Körperlagen sehr deutliche Halsu ud Labyrinthreflexe auf die Vorderbeine nachweisen.

Darauf wird der Versuch fortgeführt, indem der Hirnstamm durch Frontalschnitte von vorn nach hinten schrittweise abgetragen wird (s. u. S. 242). Die Untersuchung der abgetragenen Stücke des Hirnstammes ergibt, dass "das Kleinhirn in toto entfernt ist, und dass auch die extramedullären Anteile der Kleinhirnstiele abgetragen worden sind.

Ergebnis: Bei einer dezerebrierten Katze wird das Kleinhirn vollständig exstirpiert. Danach ist sehr starke Starre der Extremitäten und des Nackens vorhanden. Durch Änderung der Kopfstellung lassen sich kräftige tonische Hals und Labyrinthreflexe auf die Muskulatur der Vorderbeine und sehr deutliche Labyrinthreflexe auf die Nackenmuskulatur nachweisen. Nach Durchschneidung des linken Octavus kommt es zu Linksdrehing und 
-wendung des Kopfes und Linksdrehung des Rumpfes. Labyrinthreflexe auf Nacken- und Gliedermuskeln und Halsreflexe auf die Gliedermuskeln sind danach noch deutlich nachweisbar.

Noch in einem anderen Versuche (Nr. VII) liessen sich nach der Kleinhirnexstirpation Labyrinthreflexe auf die Nackenmuskulatur und nach der darauf vorgenommenen linksseitigen Labyrinthausschaltung Linkswendung des Kopfes nachweisen.

\section{Versuch XXIII. 27. Februar 1914.}

Katze. Äthernarkose. Karotiden abgebunden, doppelseitige Vagotomie, Freilegung des Rückenmarkes am zwölften Brustwirbel. Temporäre Abklemmung der Vertebralarterien. Dezerebrierung. Totalexstirpation des Kleinhirns nach vorheriger Längsspaltung. Kleinhirnstiele von der Medialseite her durchtrennt.

$10 \mathrm{Uhr} 30 \mathrm{Min}$. Ende der Operation. Beginnende Starre.

11 Uhr. Seitenlage, Kopfdrehen: Bei Scheitel-unten werden beide Vorderbeine gestreckt, das obere viel kräftiger als das untere. Bei Scheitel-oben erschlaffen beide Vorderbeine, das obere stärker (Hals: und Labyrintbreflexe, letztere überwiegen).

Seitenlage, Kopfwenden: Dasselbe (Hals- und Labyrinthreflexe, letztere überwiegen).

Seitenlage, Kopfheben und -senken: Wirkungslos (kei a e Halsreflexe bei symmetrischen Kopfbewegungen).

Rückenlage, Kopfheben und -senken: Die Vorderbeine haben maximalen Strecktonus, wenn die Schnauze $45^{\circ}$ über die Horizontale gehoben ist. Der Tonus sirikt, wenn die Schnauze durch Ventralbeugen des Kopfes $z$ wischen den Vorderpfoten steht, und wenu sie durch Dorsalbeugen des Kopfes nach unten gerichtet ist (starke Lab y rinthreflexe).

Rückenlage, Kopfdrehen: Tonusabnahme in beiden Vorderbeinen, im Schädelbein mehr (Hals-and Labyrinthreflexe, letztere überwiegen).

Rückenlage, Kopfwenden: Tonusabnahme im Schädelbein, Zunahme im Kieferbein (Halsreflex).

Fussstellung, Kopfheben und -senken: Bei gehobenem Kopf Tonuszunahme, bei gesenktem Kopf Tonusabnahme der:Vorderbeinstrecker. (überwiegend $\mathbf{L}$ aby rinthreflex).

Beim Umlegen des ganzen Tieres aus der Bauch- in Rückenlage, wobei die Stellung des Kopfes zum Rumpfe vicht geändert 
wird, erfolgt eine kräftige Streckung der Vorderbeine (Labyrinthreflex).

Nach Durchschneidung des Rückenmarkes am zwölften Brustwirbel wird die ganze Untersuchungsreihe nochmals mit demselben Ergebnis wiederholt. Da sich in diesem Versuche besonders lebhafte Labyrinthreflexe ergeben hatten, werden keine weiteren Frontalschnitte durch den Hirnstamm angelegt, sondern das Tier getötet, der Hirnstamm herausgenommen und stereoskopisch photographiert (Fig. 2).

Man sieht oben das längsgespaltene exstirpierte Kleinhirn, unten den Hirnstamm. Der Dezerebrierungsschnitt geht durch die Mitte der vorderen Vierhügel. Die Rautengrube liegt in ganzer Ausdehnung von den hinteren Vierhügeln bis zum Calamus scriptorius frei. Rechts sieht man von oben auf die durchschnittenen rechten Kleinhirnstiele. Die Schnittfläche

Fig. 2.
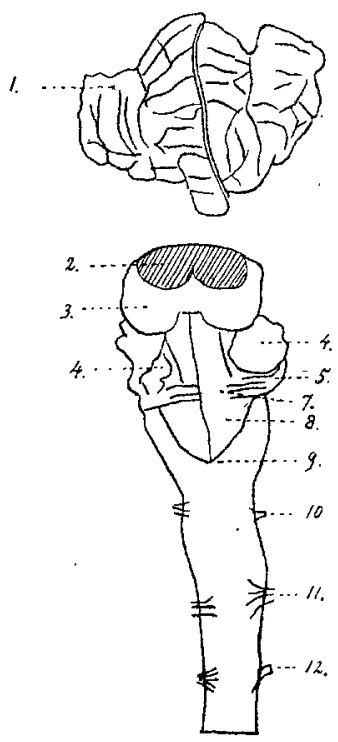

Fig. 2 a. 1 Ǩleinhirn, längsgespalten, exstirpiert. 2 Schnittfläche. 3 hintere Vierhügel. 4, 4 Schnittflächen der abgetrennten Kleinhirnstiele.

5 Nerv. octavus. 7 Striae acust. 8 Boden des vierten Ventrikels. 9 Calamus scriptorius. 10 Nerv. cervic. I. 11 Nerv. cervic. II.
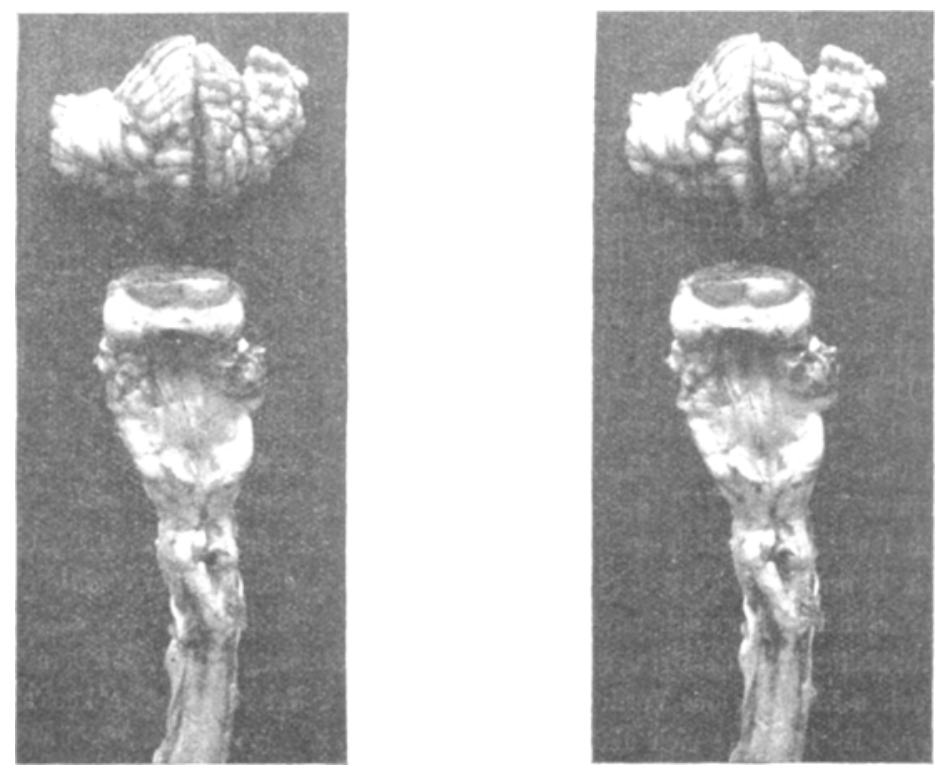
liegt $1^{1 / 2} \mathrm{~mm}$ über dem Niveau des Bodens des vierten Ventrikels. Kaudalwärts vom rechten Kleinhirnstiel schlingt sich der rechte Octavus nach der Ventralseite hinüber. Die linken Kleinhirnstiele sind innerhalb der Substanz der Medulla oblongata abgetragen. Der linke Octavusstamm war ebenfalls intakt, ist aber wegen der leichten Drehung des Präparates auf der Photographie nicht sichtbar.

Ergebnis: Bei einer dezerebrierten Katze wird das Kleinhirn vollständig exstirpiert. Danach ist gute Enthirnungsstarre vorhanden. Durch Änderung der Kopfstellung lassen sich deutliche Halsreflexe und sehr starke Labyrinthreflexe auf die Muskulatur der Vorderbeine nachweisen. Die Photographie des exstirpierten Kleinhirns und des restierenden Hirnstammes zeigt die Ausdehnung der Operation.

Aus den im vorstehenden geschilderten Versuchen, deren Ergebnisse durch die übrigen Experimente durchaus bestätigt wurden, folgt, dass bei dezerebrierten Katzen nach Entfernung des Kleinhirnes nicht nur eine vorzligliche Enthirnungsstarre vorbanden ist ${ }^{\mathbf{1}}$, sondern dass sich auch sämtliche in früheren Arbeiten von uns beschriebenen tonischen Reflexe auf die Körpermuskulatur durch Änderung der Kopfstellung auslösen lassen.

Im einzelnen wurde dieser Nachweis geführt für folgende Reflexe:

\section{A. Halsreflexe.}

1. Symmetrische Kopfbewegungen: Dorsalbeugen des Kopfes bewirkt Streckung der Vorderbeine und Beugung der Hinterbeine. Ventralbeugen des Kopfes bewirkt Streckung der Hinterbeine und Beugung der Vorderbeine ${ }^{2}$ ).

Verschiebung der unteren Halswirbel in ventraler Richtung hemmt den Strecktonus aller vier Beine.

1) Sherrington hat bereits 1898 (Journ. of Physiol. vol. 22 p. 327) angegeben: "It is significant that decerebrate rigidity sometimes persists after removal of the cerebellum, if the latter ablation be performed without any serious amount of haemorrhage."

2) Barany, Reich und Rothfeld haben in einer vorlüufigen Mitteilung (Neurol. Zentralbl. Bd. 31 S. 1139. 1912) angegeben, dass sie bei dezerebrierten Katzen oder Hunden nach unvollständiger stückweiser Entfernung des Kleinhirns auf Kopfheben Streckung der Vorderbeine und Beugung der Hinterbeine, auf Kopfsenken Beugung der Vorderbeine und Streckung der Hinterbeine beobachten konnten. Es handelte sich also um einen Halsreflex. 
2. Unsymmetrische Kopfbewegungen: Drehen und Wenden des Kopfes führt zu Zunahme des Strecktonus im vorderen und hinteren "Kieferbein" und zu Abnahme des Strecktonus im vorderen und hinteren "Schädelbein".

\section{B. Labyrinthreflexe.}

Wenn sich der Kopf in der "Maximumstellung" für die Labyrinthreflexe befindet (Scheitel unten, Unterkiefer oben, Mundspalte $45^{\circ}$ über die Horizontale gehoben), so wird dêr Strecktonus der vier Extremitäten und der Nackenheber maximal. Wenn sich der Kopf in der "Minimumstellung" befindet (Scheitel oben, Unterkiefer unten, Mundspalte $45^{\circ}$ unter die Horizontale gesenkt), so ist der Tonus dieser Muskeln minimal.

Ein Labyrinth genügt, um den Tonus der Streckmuskeln der beiderseitigen Extremitäten zu beherrschen; der Einfluss auf die Halsmuskulatur ist dagegen ein einseitiger.

Nach Ausschaltung eines Labyrinthes kommt es zur Drehung und Wendung des Kopfes nach der Seite des fehlenden Labyrinthes, zur Drehung des Rumpfes und zu einseitiger Abnahme des Tonus der Gliederstrecker.

Für das Zustandekommen aller dieser Reaktionen genügt also die Anwesenheit derjenigen Zentren, welche im Hirnstamm hinter den vorderen Vierhügeln gelegen sind. Das Vorhandensein des Kleinhirns ist dafür nicht notwendig. Nach Fortnahme des Kleinhirns sind alle diese Reflexe in unverminderter Stärke nachweisbar.

Im folgenden Abschnitt soll nun untersucht werden, welehe Teile des Hirnstammes und des Halsmarkes noch mit dem übrigen Rückenmark in Verbindung sein müssen, damit erstens die Labyrinthreflexe und zweitens die Halsreflexe zustande kommen können.

b) Verhalten der Hals- und Labyrinthreflexe nach schrittweiser Abtragung des Hirnstammes und des Halsmarkes.

Dass nach Abtragung der hinteren Vierhügel die Hals- und Labyrinthreflexe auf die Gliedermuskeln noch unverändert erhalten sind, ergibt sich aus folgendem Versuchsprotokoll.

Versuch $\mathrm{X}$. 31. Januar 1914.

Katze. Äthernarkose. Karotiden abgebunden, Vagi durchtrennt, Freilegung des Rückenmarkes am zwölften Brustwirbel. Temporäre Abklemmung der Vertebralarterien, Dezerebrierung mit Ausräumen 
des Gehirns vor den Vierhügeln. Totalexstirpation des Kleinhirns. Abtragung der Vierhügel durch einen Frontalschnitt. Dauer der Operation 15 Minuten.

10 Uhr 30 Min. Ende der Operation. Beginnende Starre der Vorderbeine.

10 Uhr 48 Min. Starre der Vorderbeine deutlich (nicht maximal), der Hinterbeine nicht deutlich.

Seitenlage, Kopfdrehen: Tonuszunahme im vorderen Kieferbein, Abnahme im vorderen Schädelbein (Halsreflex).

Seitenlage, Kopfheben und -senken: wirkungslos.

Fussstellung, Kopfheben und -senken: Kopfheben bewirkt Streckung, Kopfsenken bewirkt Beugung der Vorderbeine (La by rinth oder Halsreflexe).

Fussstellung, Kopfdrehen bei erhobener Schnatize: Tonuszunahme im vorderen Kieferbein, Abnahme im vorderen Schädelbein (Hals reflex).

Fussstellung, Kopfwenden bei horizontaler Mundspalte: Tonuszunahme im vorderen Kieferbein, Abnahme im vorderen Schädelbein (Halsreflex).

Rückenlage, Kopfdrehen: Tonusabnahme beider Vorderbeine (Labyrinthreflex).

Beim Umlegen des ganzen Tieres aus der Fussstellung in Rückenlage, ohne dass dabei die Stellung des Kopfes zum Rumpfe geändert wird, erfolgt Streckung der Vorderbeine (Labyrinthreflex).

Hängelage mit Kopf-unten, Kopfheben und -senken: Geringer Vorderbeintonus, wenn die Schnauze gerade nach untẹn hängt. Tonuszunahme, wenn die Schnauze durch Ventralbeugen des Kopfes zwischen die Vorderbeine zu stehen kommt (Labyrinthreflex).

Vertebra-prominens-Reflex: sehr deutlich.

11 Uhr. Starre der Vorderbeine deutlich, aber nicht maximal, Starre der Hinterbeine deutlich.

11 Uhr 10 Min. Rückenlage, Kopfheben und -senken: Die Vorder- und Hinterbeine haben maximalen Tonus, wenn die Mundspalte $45^{\circ}$ über die Horizontale gehoben ist. Wird der Kopf dorsalwärts gebeugt, bis die Schnauze vertikal nach unten sieht, so nimmt der Strecktonus in allen vier Beinen ab (Laby rinthreflex a u Vorder-und Hinterbeine).

Sektion: Kleinhirn fehlt vollständig. Frontalsehnitt geht genau hinter den Vierhügein, so dass diese total abgetrennt sind. Nucleus 
dentatus fehlt beiderseits. Der Deiterskern ist beiderseits intakt (Winkler).

Firgebnis: Bei einer dezerebrierten Katze sind nach Totalextirpation des Kleinhirns und Abtrennung der Vierhügel durch Änderung der Kopfstellung kräftige Hals. und Labyrinthreflexe auf die Gliedermuskeln nachawveisen.

In dem soeben geschilderten Versuche war die Enthirnungsstarre nicht sehr hochgradig; in drei anderen ebenso ausgeführten Experimenten war dagegen eine kräftige Starreder Extremitäten und der Nackenmuskulatur vorbanden.

Die drei folgenden Versuchsprotokolle zeigen nun übereinstimmend, dass man den Hirnstamm bis dicht vor dem Eintritt der Nervi octavi abtragen kann, ohne die Labyrinthreflexe (und natürlich auch die Halsreflexe) zum Verschwinden zu bringen.

\section{Versuch XIV. 6. Februar 1914.}

Katze, 1300 g. Äthernarkose. Abbinden der Karotiden, Durchtrennung der Vagi, Freilegung des Rückenmarkes am zwölften Brustwirbel, Dezerebrieren unter temporärer Abklemmung der Vertebralarterien, Totalexstirpation des Kleinhirns und der Seitenteile in einem Stück. Dauer der ganzen Operation 15 Minuten, der Kleinhirnexstirpation 5 Minuten. Direkt danach 10 Uhr 20 Min. beginnende Enthirnungsstarre.

10 Uhr 30 Min. Starke Starre aller vier Beine. Die Prüfung der Reaktionen auf Veränderung der Kopfstellung ergibt, dass das Tier an den Vorder- und Hinterbeinen überwiegende Halsreflexe hat, dass daneben aber sichere Labyrinthreflexe nachweisbar sind.

11 Uhr 15 Min. wird ein Frontalschnitt direkt hinter den Vierbügeln ausgeführt. Danach sind deutliche Hals- und Labyrinthreflexe vorhanden.

11 Uhr 25 Min. wird ein Frontalschnitt direkt oralwärts von der Eintrittsstelle der Nervi octavi geführt. Darauf wird durch Inspektion festgestellt, dass beide Octavi intakt geblieben sind. Die Prüfung auf tonische Kopfreflexe ergibt folgendes:

Seitenlage, Kopfdrehen: Tonuszunahme im Kieferbein, Abnahme im Schädelbein, unteres Bein reagiert etwas schwächer (überwiegend Halsreflexe, geringere Labyrinthreflexe). 
Rückenlage, Heben und Senken: Stärkster Strecktonus der Vorderbeine, wenn Schnauze $45^{\circ}$ über die Horizontale gehoben. Tonus sinkt deutlich, wenn der Kopf dorsal gebeugt wird, bis die Schnauze nach unten sieht; er sinkt maximal, wenn der Kopf stark ventral gebeugt wird, bis die Schnauze zwischen den Vorderbeinen steht (Labyrinthreflexe).

Rückenlage, Kopfdrehen: Tonusabnahme in beiden Vorderbeinen, im Schädelbein stärker (Labyrinth- und Halsreflexe).

Hängelage mit Kopf-unten, Kopfheben und -senken: Der Vorderbeintonus ist am geringsten, wenn die Schnauze senkrecht nach unten hängt; er nimmt zu, wenn durch Ventral- oder Dorsalbeugen die Mundspalte über die Horizontale gehoben wird. Die stärkere Tonuszunahme erfolgt beim Ventralbeugen (L abyrinthreflexe).

Der Versuch wird fortgesetzt, indem weitere Frontalschnitte durch den Hirnstamm angelegt werden (s. u. S. 246).

Die Besichtigung der abgetrennten, in Formol konservierten Stücke des Hirnstammes ergibt, dass das Kleinhirn in einem Stücke in toto exstirpiert ist. Die Kleinhirnstiele sind genau im Niveau des Bodens des vierten Ventrikels durchtrennt. Der 11 Uhr 25 Min. ausgeführte Frontalsehnitt geht rechts $2 \mathrm{~mm}$, links $1 \mathrm{~mm}$ vor dem Eintritt der Nervi octavi durch die Tubercula acustica. Vom Kleinhirn und seinen Stielen steht nichts mehr.

Ergebnis: Bei einer dezerebrierten Katze wird nach Extirpation des Kleinhirns ein Frontalabschnitt durch den Hirnstamm unmittelbar vor dem Eintritt der Octavi gemacht. Danach sind durch Kopfdrehen sowohl Hals- als auch Labyrinthreflexe auf die Vorderbeine auszulösen.

\section{Versuch XVII. 16. Februar 1914.}

Fortsetzung des auf S. 234 angeführten Protokolles. (Nach Exstirpation des Kleinhirns sind Hals- und Labyrinthreflexe auf die Vorderbeine und Labyrinthreflexe auf den Nacken nachweisbar. Nach Durchtrennung des linken Octavus kommt es zu Linksdrehung und -wendung dès Kopfes und Linksdrehung des Rumpfes. Halsund Labyrinthreflexe auf die Glieder bleiben vorbanden.)

11 Uhr 10 Min. Frontalschnitt dicht vor dem Eintritt der Nervi octavi. Danach deutliche Starre der Vorderbeine.

Seitenlage, Kopfdrehen: Tonuszunahme im Kieferbein, Abnahme im Schädelbein (Halsreflex). 
Beim Umlegen aus der Fussstellung in Rückenlage, wobei die Stellung des Kopfes zum Rumpfe nicht verändert wird, erfolgt kräftige Streckung der Vorderbeine und Retraktion des Nackens. Linkes Vorderbein bleibt dabei (als Folge der einseitigen Octavusdurchschneidung) etwas schlaffer als das rechte (Labyrinthreflexe auf Vorderbeinstrecker und Nackenmuskeln).

Bei Hängelage mit Kopf-unten ist die Linksdrehung des Kopfes noch deutlich (Wirkung des einseitigen Labyrinthausfalles auf die Halsmuskeln).

Der Versuch wird fortgesetzt, und weitere Frontalschnitte werden ausgeführt.

Die Besichtigung der abgetrennten, in Formol konservierten Stücke des Hirnstammes ergibt, dass das Kleinbirn samt den extramedullären Teilen der Kleinhirnstiele totol exstirpiert ist. Der 11 Uhr 10 Min. angelegte Frontalschnitt geht beiderseits hinter den Stümpfen der Kleinhirnstiele, links $2 \mathrm{~mm}$ vor dem Eintritt des Octavus, so dass das Tuberculum acusticum zum Teil stehen geblieben ist, rechts $1 \mathrm{~mm}$ vor dem Eintritt des Octavus durch das Tuberculum acusticum hindurch.

Ergebnis: Bei einer dezerebrierten Katze wird das Kleinhirn exstirpirt und der linke Octavus durchschnitten. Darauf wird ein Frontalschnitt dicht vor dem Eintritt der Octavi gemacht. Danach sind noch Hals- und Labyrinthreflexe auf die Vorderbeine, Labyrinthreflexe auf die Nackenmuskeln und die infolge der einseitigen Octavusdurchschneidung eintretende Kopfdrehung nachweisbar.

\section{Versuch XXII. 24. Februar 1914.}

Katze. Äthernarkose. Karotiden abgebunden, Vagi durchtrennt. Freilegung des Rückenmarkes am zwölften Brustwirbel. Temporäre Abklemmung der Vertebralarterien, Dezerebrierung. Totalexstirpation des Kleiuhirns nach vorheriger Längsspaltung. Ende der Operation 9 Uhr 55 Min. Darauf sofort gute Starre der vier Beine, Spontanatmung, Lidreflex.

$10 \mathrm{Uhr} 20 \mathrm{Min}$. Untersuchung auf Kopfreflexe ergibt das Vorhandensein von kräftigen Hals-und Labyrinthreflexen auf die Vorderbeine.

10 Uhr 45 Min. Frontalschnitt dicht vor dem Eintritt der Octavi.

10 Uhr 55 Min. Durchschneidung des Rückenmarkes am zwölften Brustwirbel. Vortreffliche Starre der Vorderbeine. 

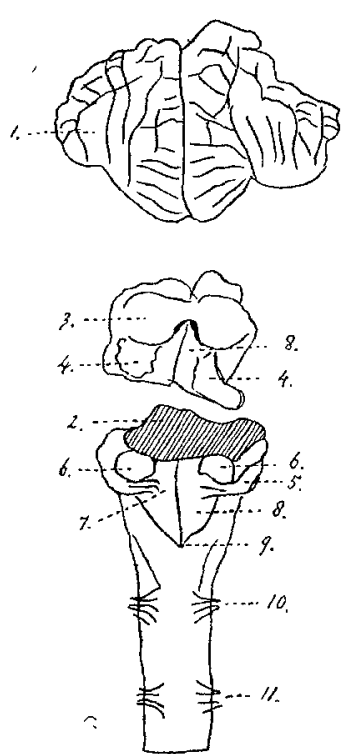

Fig. 3 a. 1 Kieinbirn, längsgespalten, exstirpiert. 2 Schnittfläche des Frontalschnittes vor dem Octarusursprung. 3 Hintere Vierhügel. 4,4 Schnittflächen der al)getrennten Kleinhirnstiele. 5 Nervus octavus. 6,6 Tubercula acustica. 7 Striae acusticae. 8,8 Boden des vierten Ventrikels. 9 Calamus scriptorius. 10 Nerv. cervic. I. 11 Nerv. cervic. II.

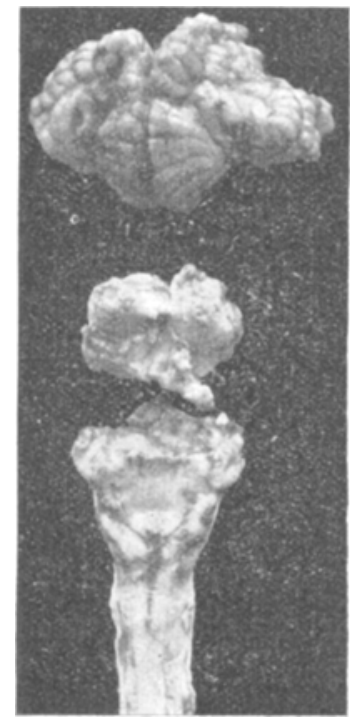

Seitenlage, Kopfdrehen: Wenn Scheitel unten, werden beide Beine gestreckt, wenn Scheitel oben, werden bëide Beine gebeugt (Labyrinthreflexe).

Rückenlage, Kopfheben und senken: Der Strecktonus der Vorderbeine ist maximal, wenn die Schnauze $45^{\circ}$ über die Horizontale gehoben ist; er nimmt bei Dorsalbeugung des Kopfes (Schnauze nach unten) und bei starker Ventralbeugung des Kopfes (Schnauze zwischen den Vorderbeinen) ab (Labyrinthreflexe).

Beim Umlegen des Tieres aus der Fussstellung in Rückenlage, wobei die Stellung des Kopfes zum Rumpfe nicht geändert wird, erfolgt starke Tonuszunahme der Vorderbeine (Labyrinthreflex).

Rückenlage, Kopfwenden: Tonuszunahme im Kieferbein, Abnahme im Schädelbein (Halsreflex).

Fussstellung, Kopfdrehen bei erhobener

Fig. 3.

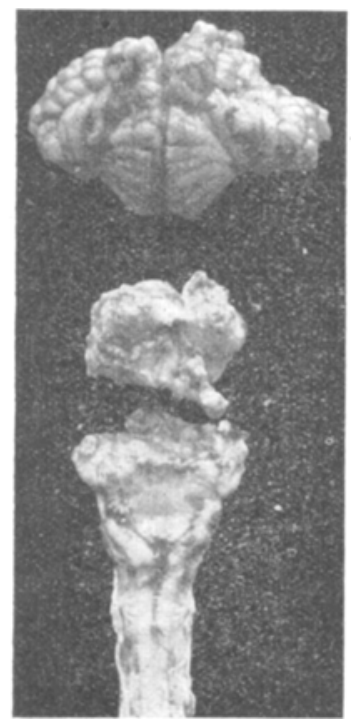


Schnauze: Tonuszunahme im Kieferbein, Abnahme im Schädelbein (Halsreflex).

Fussstellung, Kopfwenden bei horizontaler Mundspalte: Tonuszunahme im Kieferbein, Abnahme im Schädelbein (Halsreflex).

Sektion: Beide Octavi intakt. Der Frontalschnitt geht links gerade vor dem Tuberculum acusticum, rechts durch das vordere Drittel des Tuberculum acusticum. Es ist abgetrennt das ganze Kleinhirn und der ganze extramedulläre Teil der Corpora restiformia. Auf der linken Seite ist derjenige Teil des Deiters'schen Kernes stehengeblieben, der der Deitero-spinalen Babn den Ursprung gibt (genauer, der bei Hemisektion der unteren Oblongata alle Zellen verliert). Rechts ist der kleinere Teil dieses letzteren Kernes entfernt, der grössere Teil steht noch (W inkler).

Das exstirpierte Kleinhirn, das abgetrennte Stück, welches die hinteren Vierhügel, den vorderen Teil des vierten Ventrikels und die Stümpfe der Kleinhirnstiele umfasst, sowie das übrigbleibende Stück des Hirnstammes, welches zum Zustandekommen der Halsund Labyrinthreflexe genügte, werden stereoskopisch photographiert (Fig. 3). Man sieht die hintere Hälfte des Bodens vom vierten Ventrikel, die Ursprungsstelle der Octavi, den Stamm des rechten Octavus, beide Tubercula acustica, soweit sie nicht abgetrennt sind, und die Schnittfläche des Frontalschnittes.

Ergebnis: Bei einer dezerebrierten Katze wird nach Exstirpation des Kleinhirns ein Frontalschnitt durch den Hirnstamm dicht vor dem Eintritt der Octavi angelegt. Danach lassen sich durch Änderung der Kopfstellung noch kräftige Hals- und Labyrinthreflexe auf die Vorderbeine auslösen.

Wie zu erwarten ist, sind nach allen Frontalschnitten, welche hinter dem Eintritt der Octavi durch den Hirnstamm geführt werden, die Labyrinthreflexe auf die Glieder und die Stammesmuskulatur erloschen. Tonische Einflüsse auf die Muskulatur dureh Änderung der Stellung des Kopfes im Raume lassen sich nicht mehr hervorrufen.

Dagegen sind interessanterweise die Halsreflexe noch erhalten, und man muss mit den Frontalschnitten ein gutes Stück weiter kaudalwärts gehen, bis man auch diese zum Verschwinden bringt. Darüber unterrichtet das folgende Versuchsprotokoll: 


\section{Versuch XIV. 9. Februar 1914.}

(Fortsetzung des Protokolls von S. 241). Bei dieser Katze war nach Exstirpation des Kleinhirns ein Frontalschnitt direkt vor dem Eintritt der Oetavi gemacht worden. Danach liessen sich noch deutliche Hals- und Labyrinthreflexe durch Änderung der Kopfstellung auf die Vorderbeine auslösen (s. 0. S. 241 u. 242).

11 Uhr 35 Minuten. Frontalschnitt hinter den Octavis, $2^{1 / 2} \mathrm{~mm}$ vor dem Calamus scriptorius. Danach sind alle Labyrinthreflexe erloschen. Weder bei Kopfdrehen in Seitenlage noch bei Heben, Senken und Kopfdrehen in Rückenlage lässt sich eine Spur von Labyrinthreflexen nachweisen. Auf Kopfdrehen und Kopfwenden in Seitenlage treten vielmehr reine Halsreflexe (Tonuszunahme im Kieferbein und Abnahme im Schädelbein) ein. In Rückenlage lassen sich durch Heben und Senken des Kopfes ebenfalls nur Halsreflexe auslösen (Zunahme des Strecktonus der Vorderbeine bei Dorsalbeugen des Kopfes, Abnahme bei Ventralbeugen). Auch bei Kopfdrehen in Rückenlage erfolgen nur Halsreflexe (Tonuszunahme im Kieferbein und Abnahme im Schädelbein). Aus diesem Grunde wird bei der Fortsetzung des Versuches die Untersuchung der Kopfreflexe nur in Seitenlage vorgenommen.

11 Uhr 45 Minuten. Frontalschnitt durch den Calamus seriptorius. Danach noch deutliche Starre der Vorderbeine. In Seitenlage treten auf Drehen und Wenden des Kopfes noch sehr deutliche Halsreflexe an den Vorderbeinen auf (Tonuszunahme im Kieferbein und Abnahme im Schädelbein). Auch der Vertebra-prominens-Reflex (Tonusabnahme der Vorderbeine bei Ventralverschiebung der untersten Halswirbel) ist sehr lebhaft.

$11 \mathrm{Uhr} 50$ Minuten. Frontalschnitt $5^{1 / 2} \mathrm{~mm}$ hinter dem Calamus scriptorius, gerade hinter dem Ursprung der ersten Cervicalwurzel. Danach ist die Starre der Vorderbeine gering. Auf Kopfdrehen in Seitenlage erfolgen aber noch vollständig deutliche, wenn auch nicht sehr starke Halsreflexe (Tonuszunahme im Kieferbein, Abnahme im Schädelbein). Ebenso treten auf Kopfwenden noch deutliche Halsreflexe ein.

11 Uhr 55 Minuten. Frontalschnitt $5^{1 / 2} \mathrm{~mm}$ weiter nach hinten, also $11 \mathrm{~mm}$ hinter dem Calamus scriptorius. Der Schnitt liegt noch $5^{1 / 2} \mathrm{~mm}$ vor dem Ursprung der zweiten Cervicalwurzel. Danach ist die Extensorstarre der Vorderbeine sehr gering. Auf Kopfdrehen 
und Kopfwenden in Seitenlage erfolgen schwache, aber doch ganz deutliche und zweifellose Halsreflexe.

12 Uhr 2 Minuten. Frontalschnitt $5 \mathrm{~mm}$ weiter nach hinten, $1 / 2 \mathrm{~mm}$ vor dem Ursprung der zweiten Cervicalwurzel. Danach ist die Starre der Vorderheine sehr gering. Durch Kopfdrehen und Kopfwenden lassen sich keine Reflexe mehr auf die Vorderbeine auslösen.

Ergebnis: Bei einer dezerebrierten Katze wird das Kleinhirn total exstirpiert und der Hirnstamm dicht vor dem Eintritt der. Octavi quer durchtrennt. Danach sind Hals- und Labyrinthreflexe auf die Vorderbeine auszulösen. Nach Durchtrennung hinter den Octavis erlöschen alle Labyrinthreflexe, dagegen bleiben die Halsreflexe auf die Vorderbeine sehr gut erhalten. Speziell wird dieses für die Reaktionen auf Drehen, Wenden, Heben und Senken des Kopfes und für den "Vertebra-prominens-Reflex" nachgewiesen. Nach einem Querschnitt durch den Calamus sind die Halsreflexe noch sehr deutlich, nach einem Schnitt durch das Halsmark hinter dem Ursprung des ersten Cervicalnerven ist die Reaktion der Vorderbeine auf Drehen und Wenden des Kopfes abgeschwächt, aber noch deutlich vorhanden und erlischt erst nach einem Schnitte, der 1/2 $\mathrm{mm}$ vor dem Ursprung von C. 2 verläuft.

Das Resultat dieres Versuches wurde in im ganzen sieben Versuchen gesichert. In allen diesen Experimenten waren nach einem Quersehnitt durch den Calamus scriptorius, also nach Entfernung des ganzen Hirnstammes und Erhaltenbleiben des Cervicalmarkes deutliche Halsreflexe durch Kopfdrehen auszulösen. In einem Versuche beteiligten sich auch die $\mathrm{Hinterbeine} \mathrm{an} \mathrm{diesen} \mathrm{Reflexen.}$

In allen Versuchen konnte das Cervicalmark bis an den Ursprung von C. 1 abgetrennt werden, ohne dass die Halsreflexe auf Kopfdrehen erloschen. Wurde das Halsmark zwischen C. 1 und C. 2 durchtrennt, so wurde in einem Versuche die Reaktion der Vorderbeine aufgehoben; in drei Versuchen dagegen war sie noch deutlich, wenn auch abgeschwächt erhalten. Durchtrennung im Niveau von C. 2 oder hinter C. 2 hob die Halsreflexe auf Drehen und Wenden des Kopfes auf. Nur in einem Versuche war es nicht mit absoluter Sicherheit zu entscheiden, ob nach einem gerade hinter dem Ursprung von C. 2 geführten Querschnitt noch eine minimale Grenzreaktion vorhanden war, die dann erst nach einem Schnitt hinter C. 3 schwand. Jedenfalls ist nach meinen Erfahrungen in der grossen Mehrzahl der 
Fälle ein Querschnitt hinter C. 2 hinreichend, um die Halsreflexe auf Kopfdrehen und -wenden zum Versehwinden zu bringen.

Die in diesem Abschnitte geschilderten Versuche lehren demnach, dass zum Zustandekommen der tonischen Reflexe, welche durch Änderung der Kopfstellung auf die Muskulatur der Gliedmaassen und des Halses ausgelöst werden, das Rückenmark und der Hirnstamm nur so weit nach vorn erhalten sein müssen, dass die für die Auslösung der Reflexe notwendigen afferenten Bahnen ungeschmälert eintreten können. Die betreffenden Zentren, soweit sie für das Zustandekommen dieser Reflexe notwendig sind, reichen also nicht weiter oralwärts als die Eintrittszone dieser afferenten Bahnen. Ein Querschnitt 1-2 mm vor dem Eintritt der Nervi octavi, welcher Vierhügel, vordere Hälfte der Rautengrube, Kleinhirn und Kleinbirnstiele abtrennt und das Rückenmark nur noch in Verbindung lässt mit der Medulla oblongata bis zu den Tubercula acustica, stört das Eintreten von Hals- und Labyrinthreflexen auf die Gliedermuskulatur und von Labyrinthreflexen auf die Halsmuskulatur überhaupt nicht. Auch die Kopfdrehung nach Durchschneidung des einen Octavus tritt noch deutlich ein. Daraus ergibt sich, dass zum Zustandekommen dieser Reflexe weder das Kleinhirn noch die Kleinhirnstiele noch die Vierhügel und der vordere Teil der Rautengrube erforderlich sind. Wird die Eintrittszone der Octavi mit abgetrennt, so sind die Labyrinthreflexe aufgehoben, aber die Halsreflexe bleiben erhalten und lassen sich durch Drehen, Wenden, Heben und Senken des Kopfes in typischer Weise auf Vorder- und Hinterbeine auslösen. Auch der sogenannte Vertebra-prominens-Reflex ist erhalten. Man kann die ganze Medulla oblongata entfernen, ohne dass die Halsreflexe aufhören. Entfernt man das erste Cervicalsegment, so werden sie schwächer, erlöschen aber in den meisten Fällen erst, wenn auch das zweite Cervicalsegment entfernt wird ${ }^{1}$ ). Hiernach lässt sich mit Bestimmtheit, wenigstens für die Halsreflexe, welche durch Drehen und Wenden des Kopfes ausgelöst werden, behaupten, dass zu ihrem Zustandekommen Kerne der Oblongata nicht mehr erforderlich sind. Der Deiters'sche Kern, die absteigende Octavuswurze] mit den sie

1) Nach den in der vorhergehenden Arbeit von Storm van Leeuwen und mir geschilderten Versuchen gehen die afferenten Bahnen für die Halsreflexe bei der Katze durch die drei obersten cervicalen Hinterwurzeln (s. o. S. 157). 
begleitenden Nervenzellen usw. können nicht die Zentren für die Halsreflexe abgeben. Diese tonischen Halsreflexe, welche nach den Versuchen von de Kleijn und mir unter Umständen monatelang unvermindert andauern können, müssen ihre notwendige anatomische Grundlage im Rückenmarke selber besitzen.

Die Zentren für die Reflexe von den Labyrinthen auf die Augen bleiben bei dieser Untersuchung füglich ausser Betrachtung, weil wegen des weiter nach vorne gelegenen Ursprunges der Augenmuskelnerven diese Reflexe beim dezerebrierten Tiere nicht eintreten.

\section{Zusammenfassung der Ergebnisse.}

1. Die tonischen Hals- und Labyrinthreflexe auf die Extremitäten- und Nackenmuskeln, welche sich bei Katzen durch Änderung der Kopfstellung auslösen lassen, sowie die Kopfdrehung, welche nach einseitiger Durchschneidung des Octavus auftritt, bleiben unverändert erhalten, wenn man die Tiere dezerebriert, ihnen das ganze Kleinhirn und die Vierhügel exstirpiert und den Hirnstamm bis dicht vor dem Ursprung der Octavi durch einen Frontalschnitt abtrennt.

2. Entfernt man ausserdem die Eintrittszone der Octavi, so hören die Labyrinthreflexe auf; aber die Halsreflexe auf die vier Beine, welche durch Drehen, Wenden, Heben und Senken des Kopfes ausgelöst werden, bleiben erhalten. Man kann die ganze Medulla oblongata entfernen, ohne das Auftreten der Halsreflexe zu hindern. Nach Entfernung des obersten Cervicalsegmentes werden die Halsreflexe abgeschwächt, nach Entfernung des zweiten Halssegmentes fallen sie fort.

\section{Zusatz bei der Korrektur. Von}

J. S. Beritoff (St. Petersburg) und R. Magnus.

In der vorhergehenden Arbeit von Magnus war die Hauptaufgabe, das Verhalten der tonischen Hals- und Labyrinthreflexe nach Kleinhirnexstirpation und schrittweiser Abtragung des Hirnstammes zu untersuchen. Dabei konnte es nicht ausbleiben, dass auch Beobachtungen über das Verhalten der Enthirnungsstarre nach diesen Eingriffen angestellt wurden, über welche nebenbei berichtet wurde. Es sei uns 
daher gestattet, einige Bemerkungen an eine während der Drucklegung dieser Arbeit erschienene Mitteilung von L. H. Weed: Observations upon decerebrate rigidity (Journ. of Physiol. vol. 48 p. 205. 1914) zu knüpfen. W e ed exstirpierte bei dezerebrierten Katzen nachträglich das Kleinhirn, indem er es vom Boden des vierten Ventrikel abhob und die Stiele durchtrennte. Danach schwand die Enthirnungsstarre durchschnittlich innerhalb von 20 Minuten. Nur in einem Versuche blieb die Starre erhalten. In einem anderen Versuche wurde das Kleinhirn zuerst exstirpiert und danach dezerebriert; auch hierbei kam es nur für 1-2 Minuten zur Starre, und das Tier wurde danach schlaff. Bei einer Katze wurde ausserdem das Kleinhirn 4 Wochen vor der Dezerebrierung exstirpiert, an welche sich anfangs Starre anschloss, die im Verlaufe von $2^{1 / 2}$ Stunden schwand. Weed gibt ferner an, dass Durchtrennung des Hirnstammes direkt hinter den hinteren Vierhügel spätestens nach 5 Minuten von völligem Verluste der Enthirnungsstarre gefolgt sei. Aus diesen Versuchen schliesst Weed, dass das Hauptzentrum für die Enthirnungsstarre im Mittelhirn und höchstwahrscheinlich im Nucleus ruber liegt, und dass das Kleinhirn ein sehr wichtiges, wenn nicht sogar absolut notwendiges Bindeglied für die Entstehung der Enthirnungsstarre bildet.

Diese Beobachtungen und Schlussfolgerungen von Weed werden durch die oben geschilderten Versuche von Magn u s nicht bestätigt. Vielmehr ergibt sich aus ihnen, dass sow ohl nach vollständiger Entfernung des Kleinhirns als auch nach Abtragung des Hirnstammes bis hinter die Vierhügel noch eine kräftige Enthirnungsstarre auftreten kann, und dass daher weder das Kleinhirn noch der rote Kern zu den für das Entstehen der Enthirnungsstarre n o twe nd ig e n Zentralteilen gerechnet werden können. Der Unterschied in den Versuchsergebnissen beruht vielleicht daranf, dass Weed ausser in zwei Versuchen die Kleinhirnexstirpation längere Zeit nach dem Dezerebrieren vorgenommen hat und eine weniger schonende Technik verwendete.

$\mathrm{Da}$ in einem Teil der Versuche von Magn us nach einiger Zeit weitere Abtragungen des Hirnstammes vorgenommen wurden, bei denen dann schliesslich die Starre schwand, so sei hier noch über Versuche von B erit of $f$ berichtet, welche kürzlich im hiesigen pharmakologischen Institut angestellt wurden. In diesen ergab sich, dass selbst 8 Stunden nach Dezerebrierung und vollständiger Kleinhirnexstirpation noch eine gute Starre vorhanden sein kann. In einem anderen Versuche war $5^{1 / 2}$ Stunden nach Entfernung des Kleinhirns und Abtragung des Hirnstammes bis hinter die hinteren Vierhügel noch eine gute Starre vorhanden.

Aus diesen Beobachtungen ergibt sich auch, dass das Kleinhirn nicht die Rolle für die Entstehung des "Statotonus" spielen kann, die ihm von Edinger (Über das Kleinhirn und den Statotonus. Zentralbl. f. Physiol. Bd. 26 S. 618. 1912) kürzlich zugeschrieben wurde.

Dagegen können wir die alte, schon oben erwähnte Beobachtung von Sherrington, dass nach schonender Kleinexstirpation die Enthirnungsstarre andauert, durchaus bestätigen. 